Running Head: Self-Relevance Enhances Perception

\title{
Self-Relevance Enhances the Benefits of Attention on Perception
}

C. Neil Macrae, Aleksandar Visokomogilski, Marius Golubickis, Arash Sahraie

School of Psychology, University of Aberdeen, Aberdeen, AB24 3FX, Scotland, UK

Word Count: 2998

Address Correspondence to:

Neil Macrae

School of Psychology

University of Aberdeen

Aberdeen AB24 3FX

Scotland, UK

Phone: +44 (0) 1224272245

Fax: +44 (0) 1224273426

Email: c.n.macrae@abdn.ac.uk 


\begin{abstract}
Considerable efforts have focused on elucidating the influence that self-relevance exerts on perceptual decision-making. To explore this issue further, the current research explored the extent to which stimulus applicability facilitates the benefits of covert attention on early visual processing. In two experiments, we manipulated the personal-relevance of peripheral cues (i.e., geometric shapes) that preceded the appearance of target stimuli (i.e., Gabors) and asked participants to report the orientation of the stimulus with the highest contrast. The results revealed a significant effect of self-relevance on task performance. First, compared to cues associated with a friend or stranger, self-relevant cues enhanced the apparent contrast of a stimulus. Second, the benefits of self-relevance were most pronounced when cues pertained to identities that were significant (vs. trivial or irrelevant) to observers. Together, these findings demonstrate that self-relevance potentiates the benefits of transient attention on stimulus processing.
\end{abstract}

Keywords: self, stimulus contrast, perception, attention 
Self-Relevance Enhances the Benefits of Attention on Perception

Few constructs are as influential as a sense of self. Little wonder, therefore, this topic has stimulated interest from diverse sections of the academic community. The message that emerges from this work is unequivocal, self-referential processing exerts a biasing influence on core aspects of cognitive functioning; most notably, memory and decision-making (Conway \& Pleydell-Pearce, 2000; Heatherton, Macrae, \& Kelley, 2004). Given however its pivotal psychological status, one would expect the effects of the self to extend beyond judgmental and memorial outcomes. In particular, as a potent determinant of stimulus appraisal, the self-relevance of material should exert influence much earlier in the processing stream — for example, during basic attentional operations. Recent research and theorizing by Sui, Humphreys and colleagues makes just such a claim. From the multitude of competing possibilities, attention enables individuals to prioritize a subset of information for subsequent processing. In this regard, aside from everyday objects with obvious biological or social significance (e.g., snakes, angry faces), even the transient association of arbitrary (i.e., inconsequential) stimuli with the self may be sufficient to trigger attentional prioritization (Humphreys \& Sui, 2015; Sui \& Humphreys, 2015). Accordingly, here we explored the possibility that, via its effects on covert attention, self-relevance enhances early visual processing.

Several strands of research and theorizing motivate the hypothesis that self-relevance may facilitate stimulus processing. First, it has long been argued that perception can be penetrated by cognitive factors, such as people’s beliefs, values and desires (Clark, 2013). Crucially, self-relevance is deemed to wield comparable influence (Dunning \& Balcetis, 2013). Second, recent evidence has revealed the benefits of self-relevance during perceptual decision-making (Sui, He, \& Humphreys, 2012). Specifically, after coupling arbitrary geometric shapes with person-related labels, perceptualmatching judgments are fastest and most accurate for shape-label pairs associated with the self (vs. friend or stranger). Although the specific mechanism driving this effect remains a matter of continued 
debate (e.g., Reuther \& Chakravarthi, 2017; Siebold, Weaver, Donk, \& van Zoest, 2015), Humphreys and Sui (2015) have suggested that, as powerful cues for attentional selection, self-relevant stimuli influence processing in a manner that mimics the effects of physical saliency.

The prioritization of stimuli during attentional selection provides a potential pathway through which self-relevance may enhance processing. Attention can be allocated to a position in space either by an overt eye movement (i.e., endogenous attention) or through the covert deployment of attention to that location following the presence of a peripheral cue (i.e., exogenous/transient attention, Posner, 1980). Critically in this regard, transient attention has been shown to facilitate early visual processing following the presentation of peripheral cues (Carrasco, 2011; Carrasco, Ling, \& Read, 2004; Phelps, Ling, \& Carrasco, 2006). For example, Carrasco et al. (2004) presented Gabor patches — orientated to the left or right — simultaneously on either side of a fixation point. Whereas one Gabor had a fixed contrast (i.e., standard Gabor), the contrast of the other stimulus (i.e., test Gabor) varied randomly between an upper and lower limit. The participant's task was simply to report the orientation of the Gabor with the higher perceived contrast. Importantly, when transient attention was drawn to the stimulus, participants reported the Gabor as displaying a higher contrast than was actually the case (i.e., contrast enhancement), thereby revealing that covert attention altered the sensory impression of the stimulus.

Through stimulus prioritization, self-relevance may similarly enhance the benefits of transient attention on visual processing. Specifically, via their potency in drawing attention to a cued location, contrast enhancement may be amplified when cuing stimuli have previously been associated with the self in comparison to other targets (e.g., friend, stranger). For example, it is possible that, via attentional sets, self-relevance may facilitate attentional capture for personally-meaningful stimuli, thereby enhance the processing of material appearing at adjacent spatial locations (e.g., Folk, Remington, \& Johnston, 1992). That is, if attentional capture triggers fluent processing, then the source of this fluency may be attributed to physical aspects of a stimulus, such as its perceived brightness or clarity (Jacoby, Allan, 
Collins, \& Larwill, 1988; Whittlesea, Jacoby, \& Girard, 1990). We investigated this possibility in two experiments.

\section{Experiment 1:}

\section{Does Self-Relevance Enhance the Benefits of Transient Attention?}

In a shape-label association task (Sui et al., 2012), participants initially linked geometric shapes with various social targets. Next, in a modified cuing paradigm, these shapes served as peripheral cues and their effects on contrast discrimination were assessed. We expected cue-relevance to moderate the effect of transient attention on perceptions of apparent contrast, such that contrast enhancement would be most pronounced following the presentation of a self-relevant (vs. friend or stranger) cue.

\section{Method}

\section{Participants and Design}

Eighteen undergraduates ( 9 females, $M_{\text {age }}=25.00, \mathrm{SD}=4.70$ ) took part in the experiment, for which they received $£ 10 .{ }^{1}$ All participants had normal or corrected-to-normal visual acuity. Informed consent was obtained from participants prior to the commencement of the experiment and the protocol was reviewed and approved by the Ethics Committee at the School of Psychology, University of Aberdeen. The experiment had a single factor (Cue Association: self or friend or stranger) repeated measures design.

\footnotetext{
${ }^{1}$ Based on a large effect size (Sui et al., 2012), $\mathrm{G}^{*}$ Power $\left(\eta_{\mathrm{p}}{ }^{2}=.25, \alpha=.05\right.$, power $=80 \%$ ) revealed a requirement of 17 participants.
} 


\section{Stimulus Material and Procedure}

Participants were greeted by an experimenter and told they would be performing a perception task. The experiment had two phases. Following Sui et al. (2012), the first phase comprised a computerbased learning task in which participants were required to associate specific geometric shapes (i.e., circle, triangle, square) with 3 targets: self, best friend, and an unfamiliar stranger. Prior to the task, participants were asked to name their best friend. Each participant saw a title screen for $5 \mathrm{~s}$, followed by instructions indicating that they would be presented with 3 sets of shape-target pairs and their task was to form associations between the items (i.e., learn the shape that represented each target). The shapetarget pairs were presented for $60 \mathrm{~s}$ and during the learning phase the shapes were identified only by name. Shape-target associations were counterbalanced across the sample.

Next, participants performed a modified contrast sensitivity task. The stimuli were presented on a 32-inch monitor, with a screen resolution of 1920 x 1080 pixels and a $100 \mathrm{~Hz}$ refresh rate, controlled via a specialized graphics card (Display++, Cambridge Research Systems, UK). Stimuli were generated, and their presentation controlled, via purpose-built code using MatLab (version R2012a) and the Psychophysics Toolbox (version 3.0.12, Brainard, 1997) running on a MacBook Pro (Mac OS X version 10.9.5). Participants viewed the display at a distance of $58 \mathrm{~cm}$ from the monitor, with their heads stabilized by a head and chin rest. Luminance values were calibrated using a luminance meter (Konica Minolta, Tokyo, Japan). Background luminance was $12 \mathrm{~cd} / \mathrm{m}^{2}$ and a black fixation cross $\left(0.5^{\circ} \mathrm{x} 0.5^{\circ}\right)$ remained on the screen, except during periods of rest. Shape cues and Gabors (i.e., standard \& test) were presented at $4^{\circ}$ of horizontal eccentricity on each side of the fixation cross. Shape cues were $2^{\circ} \times 2^{\circ}$ black outlines of a triangle, circle, and square that appeared immediately above ( $2^{\circ}$ offset) the location of the target stimulus. Targets were Gabor patches (sinusoidal gratings enveloped by a Gaussian filter, $2^{\circ}$ diameter). The Michelson contrast of the standard Gabor remained constant at $8 \%$, whereas the contrast of the test Gabor varied from $2 \%$ to $16 \%$ in $2 \%$ increments. The Gabors were independently tilted $45^{\circ}$ to the left or right and were either 4 or 6 cycles per degree in spatial frequency, with both 
standard and test having the same spatial frequency. The cue shape, whether the standard or test were cued, the side of the display on which the cue was presented, the test contrast, and the Gabor tilt and spatial frequency were all counterbalanced across the trials.

In total, participants completed 1152 experimental trials, with 48 trials (i.e., 24 test cued \& 24 standard cued) at each Gabor contrast. Each trial started with a randomized delay period (500-1250 ms). A shape cue was then presented for $70 \mathrm{~ms}$, followed by a blank screen for $50 \mathrm{~ms}$, after which the Gabor patches appeared for 40 ms. Participants were required to report, via a button press (i.e., left or right), the orientation of the Gabor with the higher perceived contrast (Carrasco et al., 2004). Prior to the experiment proper, participants completed a practice block consisting of 48 trials that were identical to the main block. On completion of the experiment, participants were debriefed and dismissed.

\section{Results}

For each participant, the point of subjective equality (PSE) was calculated as a function of cue association (i.e., self or friend or stranger). The PSE reveals the point at which the standard and test patches were equally likely to be selected (i.e., were equivalent in perceived contrast) by participants. If transient attention enhanced sensory impressions, then when the test Gabor is cued, subjective equality should occur at lower stimulus thresholds. The results are summarized in the psychometric functions (i.e., Weibull distributions fitted to the data for each condition) displayed in Figure 1 (Panels A, B, \& C). Differences in contrast at the PSE (Panel D) were submitted to a single factor (Cue Association: self or friend or stranger) repeated measures analysis of variance (ANOVA). This yielded a significant effect of Cue Association $\left(F(2,34)=5.93, p=.006, \eta_{\mathrm{p}}{ }^{2}=.259\right)$, such that contrast enhancement was greater for self than either friend $\left[t(17)=2.48, p=.024, d_{z}=0.60\right]$ or stranger $\left[t(17)=2.97, p=.009, d_{z}=0.72\right]$. Friend and stranger did not differ significantly $[t(17)=1.10, p=.287]$. Task performance (i.e., Gabor orientation) was equivalent in each of the cued conditions (self $=80 \%$, friend $=79 \%$, stranger $=79 \%$ ). 
Rather than impacting the appearance of the stimuli, it is possible that self-relevant cues influenced performance via the operation of a response bias. Specifically, participants preferred to respond to the stimulus location that was adjacent to the self-relevant cue. This was not the case, however. Additional analysis revealed no relationship between cue association and stimulus location $\left[\chi^{2}(2)=0.91, p=.634\right.$; trials on which the cued location was selected - self $=61 \%$, friend $=59 \%$, stranger $=58 \%)$. These results demonstrate that self-relevance enhances the benefits of transient attention on perceptions of contrast (Phelps et al., 2006). Whilst cues pertaining to self, friend and stranger elevated perceptions of stimulus contrast, this effect was most pronounced for the self-relevant cue. 

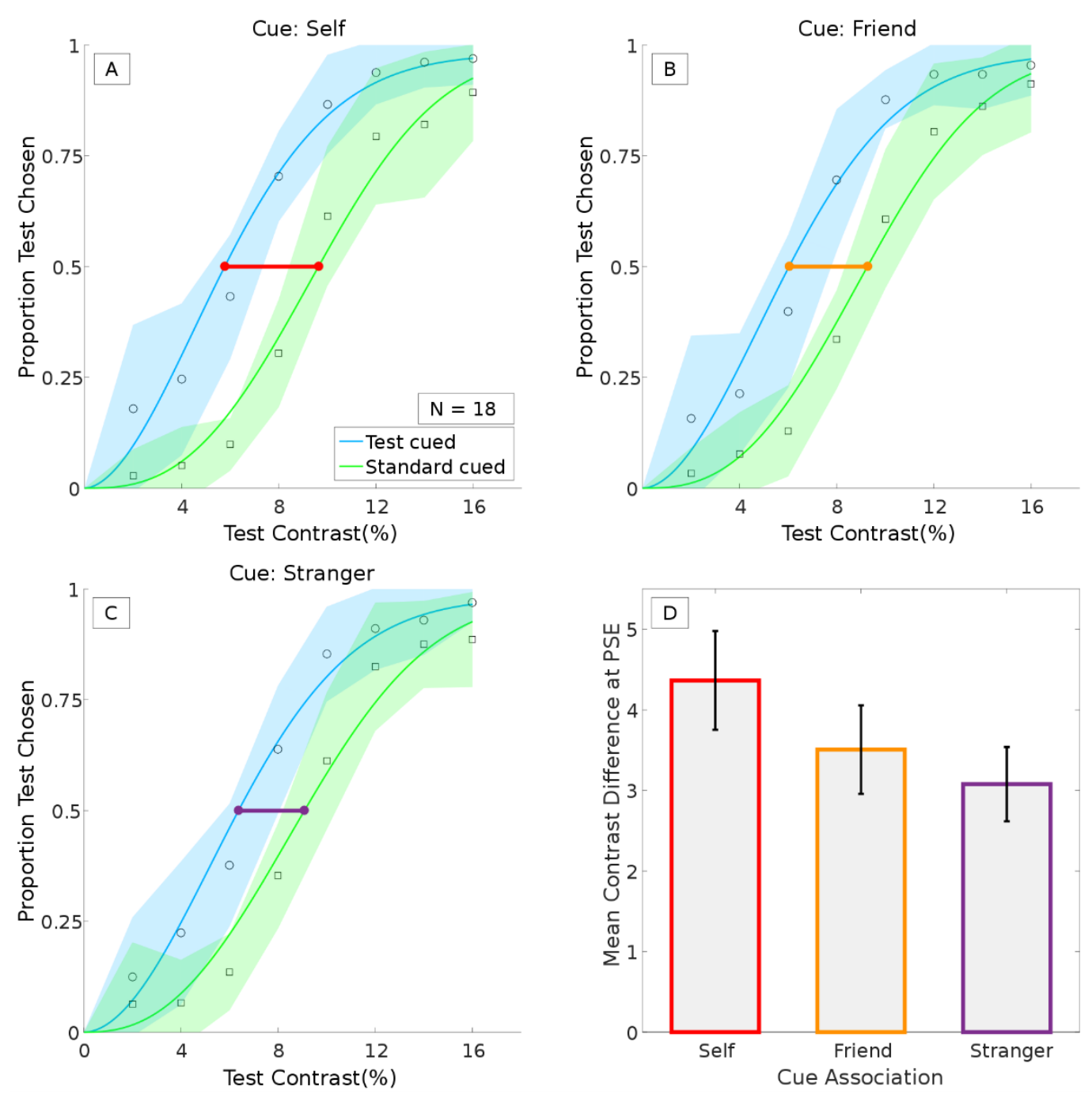

Figure 1. Psychometric functions as a function of cue association (Expt. 1). Proportion of responses in which participants reported the contrast of the test patch as higher than the standard (see Panels A, B, \& C), plotted as a function of the test Gabor's physical contrast. The standard Gabor was $8 \%$ contrast. The horizontal line intersecting both curves indicates the contrasts necessary for the test and standard Gabors to attain subjective equality (i.e., 0.5).

\section{Experiment 2:}

\section{Does Identity Strength Moderate the Benefits Effects of Transient Attention?}

While demonstrating that self-relevance enhanced the benefits of transient attention on perceptions of apparent contrast, Experiment 1 arguably failed to capture the subtle and nuanced ways in 
which the self can impact perception. Rather than comprising a unitary, monolithic entity, the self is a multifaceted construct shaped by the collective influence of prior knowledge, situational forces, and processing goals (McConnell, 2011). In particular, each person’s self-concept comprises a collection of context-dependent identities (e.g., graduate student, musician, libertarian) that vary in centrality and significance, hence processing power. Based on this representational complexity, theories of identitybased motivation contend that when a specific component of the self is activated (i.e., a person's working self), attentional resources are preferentially allocated to stimuli that bolster and enhance that identity (Coleman \& Williams, 2015). Moreover, identities that are most relevant or important to a person exert the greatest effect on stimulus processing (Oyserman, 2007). What this therefore suggests is that identity-relevance may modulate the benefits of transient attention on stimulus processing. We investigated this possibility in our second experiment.

\section{Method}

\section{Participants and Design}

Thirty undergraduates (21 females, $M_{\text {age }}=21.04, \mathrm{SD}=1.62$ ) took part in the experiment. ${ }^{2}$ All participants had normal or corrected-to-normal visual acuity. Three participants ( 2 females) failed to follow the instructions, thus were excluded from the analysis. Informed consent was obtained from participants prior to the commencement of the experiment and the protocol was reviewed and approved by the Ethics Committee at the School of Psychology, University of Aberdeen. The experiment had a single factor (Identity Strength: high or low or irrelevant) repeated measures design.

\footnotetext{
2 Based on a medium to large effect size (Coleman \& Williams, 2015), G*Power $\left(\eta_{\mathrm{p}}{ }^{2}=.15, \alpha=.05\right.$, power $\left.=80 \%\right)$ revealed a requirement of 30 participants.
} 


\section{Stimulus Materials and Procedure}

The study was identical to Experiment 1, but with an important modification. Rather than forming associations between geometric shapes and different targets, participants were required to forge links between shapes and personal identities that varied in relevance/importance. Specifically, prior to the learning phase of the experiment, each participant was required to generate and write down on a piece of paper three identities: one that was highly important to them (e.g., Bulgarian); one that weakly important to them (e.g., dancer); and another that was entirely irrelevant to them (e.g., vegetarian). These identities were then associated with the geometric shapes (i.e., circle, triangle, square). Following the shape-identity learning phase, as in Experiment 1, participants performed the contrast discrimination task. On completion of the experiment, participants were debriefed and dismissed.

\section{Results}

As in Experiment 1, the point of subjective equality (PSE) was calculated as a function of cue (i.e., identity) strength (i.e., high or low or irrelevant) for each participant. The results are summarized in the psychometric functions (i.e., Weibull distributions fitted to the data for each condition) displayed in Figure 2 (Panels A, B, \& C). Differences in contrast at the PSE (Panel D) were submitted to a single factor (Identity Strength: high or low or irrelevant) repeated measures ANOVA. This revealed a significant effect of Identity Strength $\left(F(2,52)=4.77, p=.013, \eta_{\mathrm{p}}^{2}=.155\right)$, such that contrast enhancement was greater for a high-relevance than either a low-relevance $\left[t(26)=2.45, p=.021, d_{z}=\right.$ $0.48]$ or irrelevant identity $\left[t(26)=2.84, p=.009, d_{z}=0.56\right]$. Low-relevance and irrelevant identities did not differ significantly $[t(26)=0.75, p=.458]$. Task performance (i.e., Gabor orientation) was equivalent in each of the cued conditions (high $=72 \%$, low $=71 \%$, irrelevant $=71 \%$ ). Additional analysis revealed no relationship between identity strength and stimulus location $\left[\chi^{2}(2)=0.22, p=.896\right.$; 
trials on which the cued located was selected - self $=58 \%$, friend $=57 \%$, stranger $=57 \%$, thereby ruling out the operation of a response bias during the task.

Extending Experiment 1, these results provide further evidence that self-relevance potentiates the benefits of transient attention on perceptions of apparent contrast. Whilst perceptions of stimulus contrast were elevated in all three cuing conditions, contrast enhancement was most pronounced when the cue pertained to an important (vs. inconsequential or trivial) personal identity (Oyserman, 2007). 

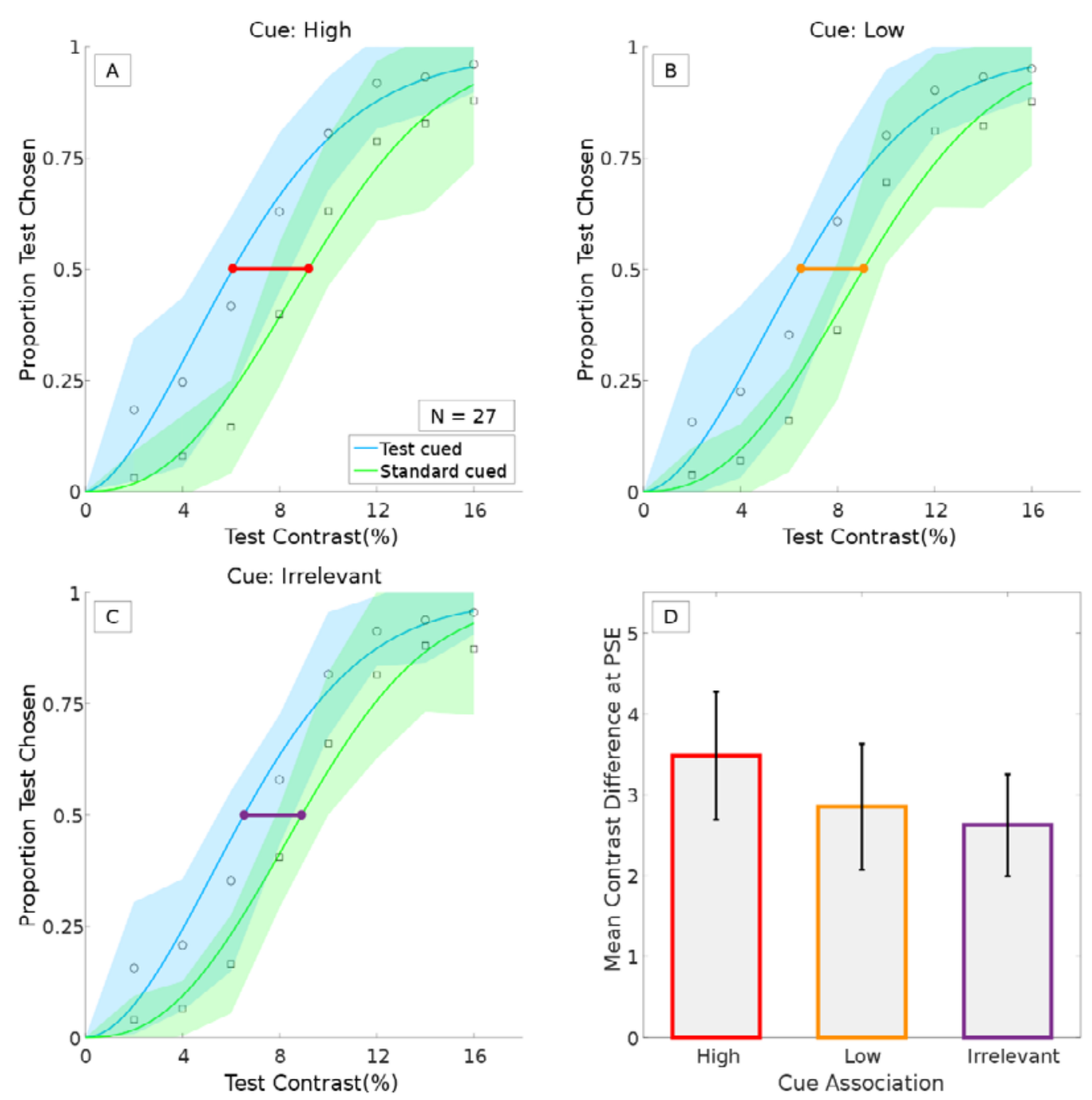

Figure 2. Psychometric functions as a function of cue association (Expt. 2). Proportion of responses in which participants reported the contrast of the test patch as higher than the standard (see Panels A, B, \& $\mathrm{C}$ ), plotted as a function of the test Gabor's physical contrast. The standard Gabor was 8\% contrast. The horizontal line intersecting both curves indicates the contrasts necessary for the test and standard Gabors to attain subjective equality (i.e., 0.5).

\section{General Discussion}

Across two experiments, the current results confirmed that self-relevance facilitates stimulus processing — notably, contrast sensitivity. First, compared to peripheral cues associated with a friend or stranger, through their effects on transient attention, self-relevant cues further enhanced perceptions of 
contrast (Expt. 1). Second, the benefits of self-relevance (i.e., contrast enhancement) were most pronounced when peripheral cues pertained to identities that were important (vs. trivial) to participants (Expt. 2). Unlike Carrasco et al. (2004), the current effects were restricted to the appearance of the stimuli, self-relevant cues did not improve task performance (i.e., identifying the orientation of the Gabors). Why this is the case is unclear, although obvious differences in the complexity of the peripheral cues that were used (i.e., a small black dot vs. geometric shapes) may contribute to these divergent results. Future research will be required to clarify this matter.

To date, research on how and when self-relevance facilitates processing has focused almost entirely on decisional efficiency during a perceptual-matching task (Sui \& Humphreys, 2015). Although this work has provided valuable insights into the dynamics of self-referential processing, questions remain regarding the extent to which self-relevance influences visual processing. Here we provided just such a demonstration. Specifically, perceived contrast was enhanced by the presence of self- and identity-relevant stimuli (i.e., top-down attentional amplification of early visual processing). As selfrelevance commonly signals the value or importance of information, the benefits of stimulus prioritization are obvious — through enhanced processing people can respond quickly and accurately to environmental threats, challenges, and opportunities. 


\section{References}

Brainard, D. H. (1997). The psychophysics toolbox. Spatial Vision, 10, 433-436.

Carrasco, M. (2011). Visual attention: The past 25 years. Vision Research, 51, 1484-1525.

Carrasco, M., Ling, S., \& Read, S. (2004). Attention alters appearance. Nature Neuroscience, 7, 308313.

Clark, A. (2013). Whatever next? Predictive brains, situated agents, and the future of cognitive science. Behavioral and Brain Sciences, 36, 181-204.

Coleman, N. V., \& Williams, P. (2015). Looking for my self: Identity-driven attention allocation. Journal of Consumer Psychology, 25, 504-511.

Conway, M. A., \& Pleydell-Pearce, C. W. (2000). The construction of autobiographical memories in the self-memory system. Psychological Review, 107, 261-288.

Dunning, D., \& Balcetis, E. (2013). Wishful seeing: How preferences shape visual perception. Current Directions in Psychological Science, 22, 33-37.

Folk, C. L., Remington, R. W., \& Johnston, J. C. (1992). Involuntary covert orienting is contingent on attentional control settings. Journal of Experimental Psychology: Human Perception and Performance, 18, 1030-1044.

Heatherton, T. F., Macrae, C. N., \& Kelley, W. M. (2004). A social brain sciences approach to studying the self. Current Directions in Psychological Science, 13, 190-193.

Humphreys, G. W., \& Sui, J. (2015). Attentional control and the self: The self-attention network (SAN). Cognitive Neuroscience, 7, 5-17. 
Jacoby, L. L., Allan, L. G., Collins, J.C., \& Larwill, L. K. (1988). Memory influences subject experience: Noise judgments. Journal of Experimental Psychology: Learning, memory, and Cognition, 14, 240-247.

Macrae, C N., Visokomogilski, A., Golubickis, M., Cunningham, W. A., \& Sahraie, A. (2017). Selfrelevance prioritizes access to visual awareness. Journal of Experimental Psychology: Human Perception and Performance, 43, 438-443.

McConnell, A.R. (2011). The multiple self-aspects framework: Self-concept representation and its implications. Personality and Social Psychology Review, 15, 3-27.

Oyserman, D. (2007). Social identity and self-regulation. In A.W. Kruglanski \& E.T. Higgins (Eds.), Social psychology: Handbook of basic principles (2dn ed., pp. 432-453). New York: Guilford Press.

Phelps, E. A., Ling, S., \& Carrasco, M. (2006). Emotion facilitates perception and potentiates the perceptual benefits of attention. Psychological Science, 17, 292-299.

Posner, M. I. (1980). Orienting of attention. Quarterly Journal of Experimental Psychology, 32, 3-25.

Reuther, J., \& Chakravarthi, R. (2017). Does self-prioritization affect perceptual processes? Visual Cognition, 25, 381-398.

Siebold, A., Weaver, M. D., Donk, M., \& van Zoest, W. (2015). Social salience does not transfer to oculomotor visual search. Visual Cognition, 23, 989-1019.

Stein, T., Siebold, A., \& van Zoest, W. (2016). Testing the idea of privileged awareness of self-relevant information. Journal of Experimental Psychology: Human Perception and Performance, 42, 303-307. 
Sui, J., \& Humphreys, G. W. (2015). The integrative self: How self-reference integrates perception and memory. Trends in Cognitive Sciences, 19, 719-728.

Sui, J., He, X., \& Humphreys, G. W. (2012). Perceptual effects of social salience: Evidence from selfprioritization effects on perceptual matching. Journal of Experimental Psychology: Human Perception and Performance, 38, 1105-1117.

Whittlesea, B. W. A., Jacoby, L L., \& Girard, K. (1990). Illusions of immediate memory: Evidence of an attributional basis for feelings of familiarity and perceptual quality. Journal of Memory and Language, 29, 716-732. 